\title{
Optimization of Transverse Oscillating Fields for Vector Velocity Estimation with Convex Arrays
}

\author{
Jensen, Jørgen Arendt
}

Published in:

Proceedings of IEEE International Ultrasonics Symposium

Link to article, DOI:

10.1109/ULTSYM.2013.0447

Publication date:

2013

Document Version

Early version, also known as pre-print

Link back to DTU Orbit

Citation (APA):

Jensen, J. A. (2013). Optimization of Transverse Oscillating Fields for Vector Velocity Estimation with Convex Arrays. In Proceedings of IEEE International Ultrasonics Symposium (pp. 1753-1756). IEEE. https://doi.org/10.1109/ULTSYM.2013.0447

\section{General rights}

Copyright and moral rights for the publications made accessible in the public portal are retained by the authors and/or other copyright owners and it is a condition of accessing publications that users recognise and abide by the legal requirements associated with these rights.

- Users may download and print one copy of any publication from the public portal for the purpose of private study or research.

- You may not further distribute the material or use it for any profit-making activity or commercial gain

- You may freely distribute the URL identifying the publication in the public portal 
Paper presented at the IEEE International Ultrasonics Symposium, Prague, Czech Republic, 2013:

\section{Optimization of Transverse Oscillating Fields for Vector Velocity Estimation with Convex Arrays}

Jørgen Arendt Jensen

Center for Fast Ultrasound Imaging,

Biomedical Engineering group, Department of Electrical Engineering, Bldg. 349, Technical University of Denmark, DK-2800 Kgs. Lyngby, Denmark 


\title{
Optimization of Transverse Oscillating Fields for Vector Velocity Estimation with Convex Arrays
}

\author{
Jørgen Arendt Jensen \\ Center for Fast Ultrasound Imaging, Department of Electrical Engineering, \\ Technical University of Denmark, DK-2800 Lyngby, Denmark
}

\begin{abstract}
A method for making Vector Flow Images using the transverse oscillation (TO) approach on a convex array is presented. The paper presents optimization schemes for TO fields for convex probes and evaluates their performance using Field II simulations and measurements using the SARUS experimental scanner. A $3 \mathrm{MHz} 192$ elements convex array probe (pitch 0.33 $\mathbf{m m}$ ) is used in both simulations and measurements. An F-number of 5 is used in transmit and two 32 element wide peaks are used in receive separated by 96 elements between peaks. Parabolic velocity profiles are simulated at beam-to-flow angles from 90 to 45 degrees in steps of 15 degrees. The optimization routine changes the lateral oscillation period $\lambda_{x}$ to yield the best possible estimates based on the energy ratio between positive and negative spatial frequencies in the ultrasound field. The basic equation for $\lambda_{x}$ gives $1.14 \mathrm{~mm}$ at $40 \mathrm{~mm}$, and $1.51 \mathrm{~mm}$ from the simulated point spread function. This results in a bias of $35 \%$ as $\lambda_{x}$ directly scales the estimated velocities. Optimizing the focusing yields a $\lambda_{x}$ of $1.61 \mathrm{~mm}$. The energy ratio is reduced from $-12.8 \mathrm{~dB}$ to -20.1 $\mathrm{dB}$ and the spectral bandwidth from $115.1 \mathrm{~m}^{-1}$ to $96.5 \mathrm{~m}^{-1} \cdot \lambda_{x}$ is maintained between 1.47 and $1.70 \mathrm{~mm}$ from $25 \mathrm{~mm}$ to $70 \mathrm{~mm}$ and is increased to $2.8 \mathrm{~mm}$ at a depth of $100 \mathrm{~mm}$. Parabolic profiles are estimated using 16 emissions. The optimization gives a reduction in std. from $8.5 \%$ to $5.9 \%$ with a reduction in bias from $35 \%$ to $1.02 \%$ at 90 degrees (transverse flow) at a depth of $40 \mathrm{~mm}$. Measurements have been made using the SARUS experimental ultrasound scanner and a BK Medical 8820e convex array transducer. Sixty-four elements was used in transmit and $2 \times 32$ elements in receive for creating a color flow map image of a flow rig phantom with a laminar, parabolic flow. At 75 degrees a bias of less than $1 \%$ was obtained.
\end{abstract}

\section{INTRODUCTION}

Vector velocity imaging using the transverse oscillation (TO) approach has recently been FDA approved for clinical use [1]. The current method employs linear array transducers, which limits the field of view and limits the method to small parts scanning. A further development with increased field of view and penetration depth is, thus, needed. This has been obtained for phased array scanning [2]. For abdominal scanning this can be attained by using convex array transducers. The paper presents an optimization scheme for TO fields for convex probes and evaluates the method's performance using Field II simulations of parabolic flow.

The TO approach introduces a transverse oscillation for estimating the lateral velocity component [3], [4]. A weakly focused field is transmitted and focused in receive with an apodization curve containing two separated peaks. Two beams are made during receive: the in-phase and the 90 degrees phase shifted quadrature beam. Fourier transforming the point spread function should thereby give a one sided spectrum for both temporal and spatial frequencies. Phase errors between the two beam results in energy appearing in the spectrum at negative spatial frequencies, which can bias the estimates of the lateral velocity component. The TO lateral wavelength $\lambda_{x}$ is predicted from the distance between peaks, scan depth, and wavelength, and determine the focusing delays to form the two beams. The simple equation underestimates $\lambda_{x}$, and simulations must be made to obtain a one-sided spectrum due to the complex interaction between focusing, apodization, and wavelength. A design procedure based on Field II simulations is suggested to reduce standard deviation and bias of the estimates. The criteria for optimality is the ratio between the energy in the right half plane to the left plane of the spectrum.

The theory behind the optimization approach is given in Section II. Optimization results are given in Section III and the approach's performance is revealed through Field II simulations in Section IV for a parabolic, laminar flow. Finally the approach is implemented on the experimental ultrasound scanner SARUS and a vector flow imaging (VFI) for a flow rig is presented along with quantitative results.

\section{THEORY AND GEOMETRICAL SET-UP}

The transverse oscillating ultrasound field is created by having a receive apodization function containing two distinct peaks [3]. The separation between the peaks determines the lateral oscillation period, $\lambda_{x}$, which is

$$
\lambda_{x}=\frac{2 \lambda D}{P_{d}},
$$

where $\lambda$ is the axial wavelength, $D$ is the depth and $P_{d}$ is the distance between the two peaks in the apodization function. The ratio $D / P_{d}$ can also be seen as the $F \#$ (F-number) for the system. A traditionally focused beam with a fairly high $F \#(5-8)$ is transmitted to generate a broad beam and two beams (in-phase and quadrature) have to be focused during transmit. The beams have to be phased shifted $90^{\circ}$ in the lateral direction to form a Hilbert transform pair, which can be used for finding both the transverse velocity magnitude and the sign of the velocity. The focusing of the two simultaneous beams therefore has to be displaced $\lambda_{x} / 4$ compared to each other at the point where the velocity estimation should take place. Note that this focusing is made in receive and therefore can be adapted as a function of depth. 

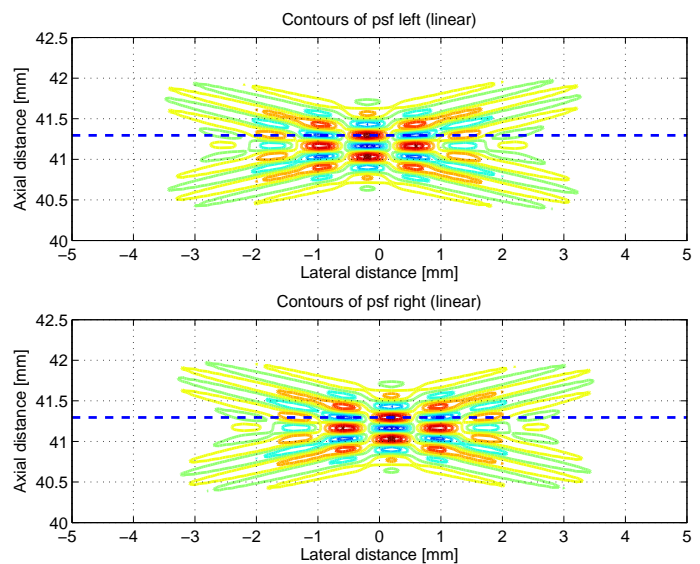

Fig. 1. Linear contour plots of the left and right point spread functions at a depth of $40 \mathrm{~mm}$.

An example of two such fields is shown in Fig. 1. The apodization function employed here has a separation between the peaks of 96 elements and uses 64 active elements in receive. A $3 \mathrm{MHz}$ convex array transducer with $\lambda$ pitch is used. It has a convex radius of $60 \mathrm{~mm}$ and an electronic transmit focus at $100 \mathrm{~mm}$. A Hamming apodization function is used during transmit.

Fig. 1 shows the resulting left and right point spread functions in a linear contour plot. The fields are displaced slightly to either the left or right compared to the center line. The two lateral PSFs nearly form a Hilbert transform pair, where the right response is the Hilbert transform of the left signal. The two fields do not exactly form a Hilbert transform pair and this results in spectral leakage from positive frequencies to negative frequencies of the two-dimensional Fourier transform of the complex transverse oscillation field. Ideally there should be no energy for negative spatial frequencies, but a peak at negative frequencies are found due to the non-ideal Hilbert relation. The $\lambda_{x}$ found by (1) is often underestimated as the formula assumes continuous wave emission, and hence focusing the pulsed field, directly at $\pm \lambda_{x} / 4$ does not result in the best Hilbert transform pair. The lateral oscillating field can be optimized by changing the focusing, the apodization, the number of active elements, the transmitted field and the number of oscillations in the field. The objective is to minimize the residual left side energy of the two-dimensional Fourier transform of the complex transverse oscillation field [5].

\section{A. Evaluation of transverse oscillation fields}

The complex field and its optimization for velocity estimation can be characterized by its estimated spatial mean frequency given by:

$$
\bar{f}_{s p}=\frac{\int_{-f_{s} / 2}^{+f_{s} / 2} \int_{-f_{s x} / 2}^{+f_{s x} / 2} f_{s p}\left|H\left(f_{t}, f_{s p}\right)\right|^{2} d f_{t} d f_{s p}}{\int_{-f_{s} / 2}^{+f_{s} / 2} \int_{-f_{s x} / 2}^{+f_{s x} / 2}\left|H\left(f_{t}, f_{s p}\right)\right|^{2} d f_{t} d f_{s p}}
$$

where $H\left(f_{t}, f_{s p}\right)$ is the Fourier transform of the complex point spread function, where the left field is the in-phase component
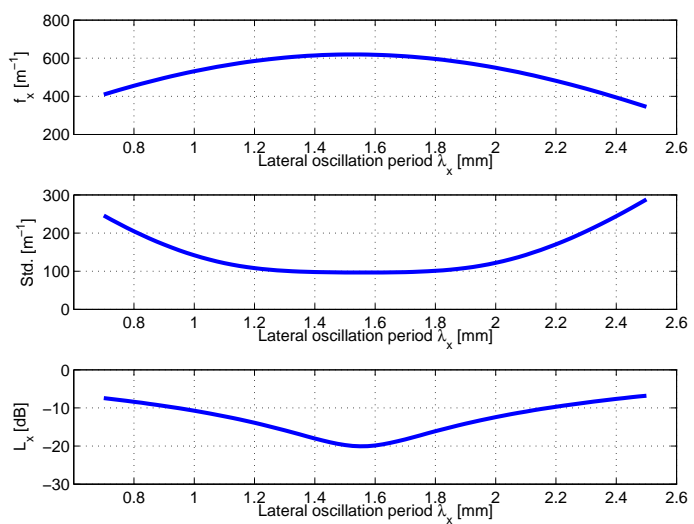

Fig. 2. Influence of a variation in the desired lateral oscillation period. The top graphs shows the obtained mean frequency, the middle graph the standard deviation of the field, and the lower graph the spectral leakage ratio.

and the right field is the quadrature component. The spatial mean frequency directly scales the velocity estimate as $\lambda_{x}=$ $1 / \bar{f}_{s p}$ in the velocity estimator. A precise knowledge of the lateral oscillation period is, thus, needed and can be estimated by (2). The lateral oscillation period also affects the receive beamformation as the left and right beam have to be shifted a quarter wavelength compared to each other.

A measure of the spectral spread is obtained by:

$$
\sigma_{\bar{f}_{s p}}^{2}=\frac{\int_{-f_{s} / 2}^{+f_{s} / 2} \int_{-f_{s x} / 2}^{+f_{s x} / 2}\left(f_{s p}-\bar{f}_{s p}\right)^{2}\left|H\left(f_{t}, f_{s p}\right)\right|^{2} d f_{t} d f_{s p}}{\int_{-f_{s} / 2}^{+f_{s} / 2} \int_{-f_{s x} / 2}^{+f_{s x} / 2}\left|H\left(f_{t}, f_{s p}\right)\right|^{2} d f_{t} d f_{s p}} \text {. }
$$

This can form the basis for the optimization of the spatial quadrature field. Minimizing $\sigma_{\bar{f}_{\text {space }}}^{2}$ will give the best result in terms of the most narrow spectrum, which directly affects the variance of the estimated lateral velocity.

Another parameter to optimize is to reduce the normalized spectral leakage ratio $L_{r}$, so that most of the energy is concentrated for positive spatial frequencies. This is calculated as

$$
L_{r}=\frac{\int_{-f_{s} / 2}^{+f_{s} / 2} \int_{-f_{s x} / 2}^{0}\left|H\left(f_{t}, f_{s p}\right)\right|^{2} d f_{t} d f_{s p}}{\int_{-f_{s} / 2}^{+f_{s} / 2} \int_{-f_{s x} / 2}^{+f_{s x} / 2}\left|H\left(f_{t}, f_{s p}\right)\right|^{2} d f_{t} d f_{s p}} .
$$

A high leakage will bias the estimate downwards. All three performance metrics are related as will be shown below.

\section{OPTIMIZATION OF THE LATERAL OSCILLATION FIELD}

A variation of the assumed lateral oscillation period and the resulting performance metrics are shown in Fig. 2, where the top graph shows the obtained mean frequency. The middle graph shows the standard deviation of the field, where a low value is beneficial as this gives the lowest variance on the estimate. The lower graph shows the spectral leakage ratio and a low ratio will give the least bias in the estimate. The best value for $\lambda_{x}$ in all graphs lies around a lateral wavelength of $1.55 \mathrm{~mm}$. 


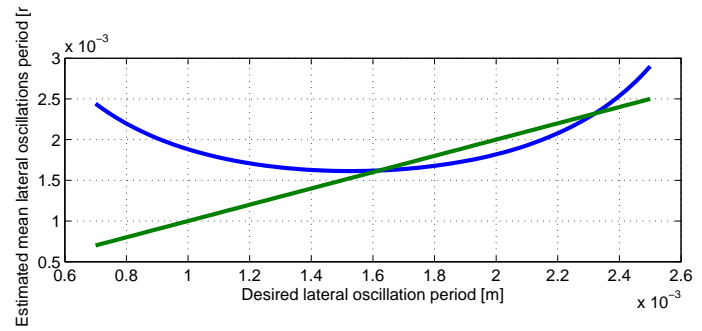

Fig. 3. The graph shows the obtained mean lateral oscillation period (blue curve) as a function of the desired period (green curve).
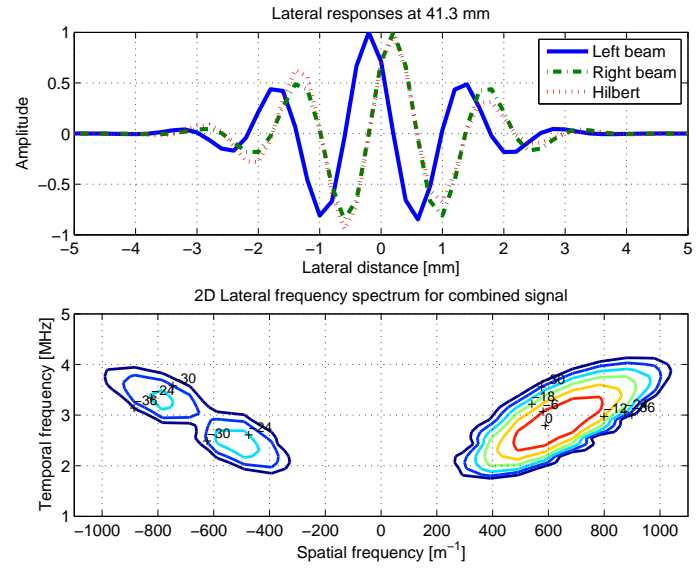

Fig. 4. The top graphs shows the lateral signals in the point spread function at the peak of the point spread function as indicated by the dashed lines in Fig. 1. The two-dimensional Fourier transform of the optimized complex transverse oscillation field is shown in the bottom graph.

Fig. 3 shows the estimated lateral wavelength found from (2) as a function of the assumed wavelength used for designing the focusing. It can be seen that setting the wavelength to $\lambda_{x}=1.55 \mathrm{~mm}$ gives a field where the desired and obtained wavelength are the same, and this also corresponds to the optimal value given in Fig. 2.

Before optimization the lateral wavelength is $1.14 \mathrm{~mm}$ calculated by (1). The spectral leakage ratio is $L_{r}=5.19 \%$ equivalent to $-12.8 \mathrm{~dB}$. The optimized lateral field has a lateral oscillations frequency of $619.6 \mathrm{~m}^{-1}$, corresponding to a lateral wavelength of $\lambda_{x}=1.61 \mathrm{~mm}$ and the fractional energy below zero frequency is reduced to $-20.1 \mathrm{~dB}$. There is, thus, a slight discrepancy between the $\lambda_{x}$ desired and the one obtained even for the optimized field. This can be compensated for by using the estimated $\lambda_{x}$ during the velocity estimation to reduce bias as demonstrated later.

The lateral oscillation signal at the peak value of the point spread function is shown in the top graph in Fig. 4. The blue curve is the left signal and the green dashed curve is the right signal. The Hilbert transform of the left signal is shown as the red dashed curve and is seen to closely match the green curve indicating that the signals form a Hilbert transform pair. The spectrum of the field is also shown in the bottom. The left area shows the reduced undesired leaked field, which has been minimized.
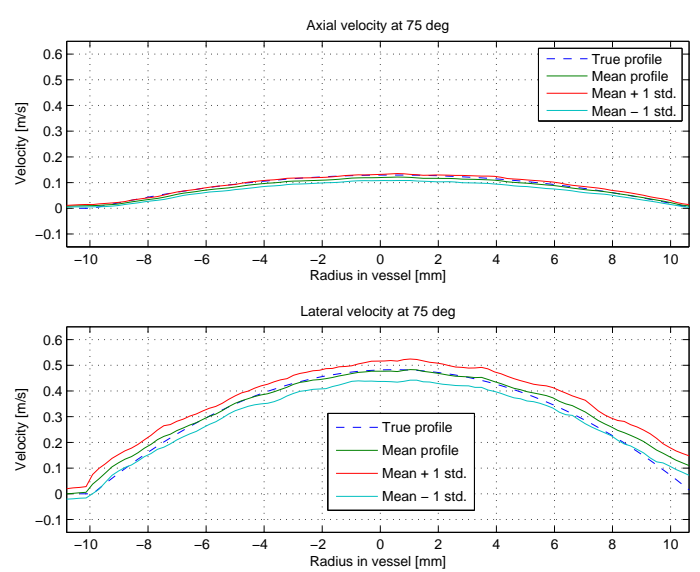

Fig. 5. Velocity profiles obtained at a beam-to-flow angle of 75 degrees.

\section{Simulation RESUlTS}

Parabolic velocity profiles have been simulated using the Field II program [6], [7] for 2,000 pulse echo lines and a vessel at a depth of $40 \mathrm{~mm}$. The velocities are estimated by the standard TO estimators derived in [5]. Sixteen emissions was used for finding one estimate yielding 125 profiles from which the mean, standard deviation, and bias was calculated relative to the true profile as shown in Fig. 5. For a beam-to-flow angle of 75 degrees the mean relative standard deviation for the transverse velocity is $5.9 \%$ and $2.1 \%$ for the axial velocity compared to the peak velocity of $0.5 \mathrm{~m} / \mathrm{s}$. The optimization gives a reduction in standard deviation from $8.5 \%$ to $5.9 \%$ with a reduction in bias from $35 \%$ to $1.02 \%$ at 90 degrees (transverse flow) at a depth of $40 \mathrm{~mm}$. When the angle changes from 90 to 45 degrees, the std. is increased from 5.9\% to $12.7 \%$ due to the increase in the axial velocity component.

\section{EXPERIMENTAL RESULTS}

The velocity estimation approach has been implemented on the SARUS experimental scanner [8]. A BK Medical 8820e transducer was employed and vector flow imaging (VFI) was interleaved with a B-mode image. An active aperture of 64 elements was used during transmit for both sequences. The focal point was at $42 \mathrm{~mm}(F \#=2)$ for the B-mode and 105.6 $\mathrm{mm}(F \#=5)$ for VFI. The transducer has 192 elements with $\lambda$ pitch and the B-mode image consisted of 129 lines. VFI was performed in 32 direction with a pitch of 4 elements and 32 emissions were made in each direction. A $6 \mathrm{~mm}$ radius tube in a circulating flow rig was scanned and the volume flow was also measured by a Danfoss Magnetic flow meter for reference. The volume flow was $112.7 \mathrm{l} / \mathrm{h}$ corresponding to a peak velocity in the vessel of $0.55 \mathrm{~m} / \mathrm{s}$. The pulse repetition frequency was $4 \mathrm{kHz}$.

The resulting velocity profiles for a measurement at $75^{\circ}$ at the center of the image is shown in Fig. 6, where the top graph shows the mean and std. of the 160 profiles for the axial velocity component. The lower graph shows the mean \pm one standard deviation for the transverse velocity component. Only 8 emissions was used for estimating the 


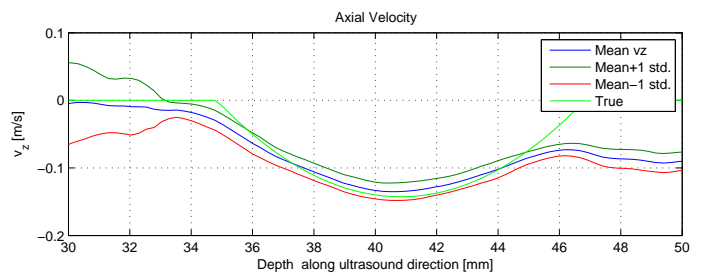

Lateral Velocity

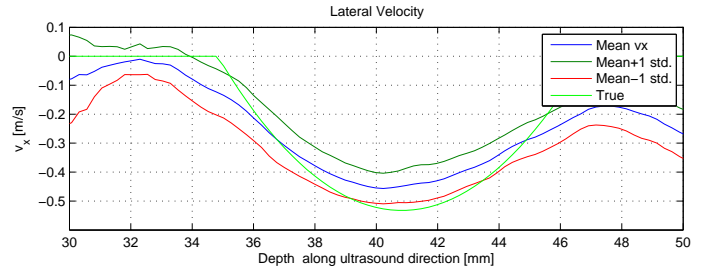

Fig. 6. Mean measured velocity profiles at a 75 degrees flow angle for the axial (top) and lateral (bottom) velocity component. The green curve indicates the true velocity profile.
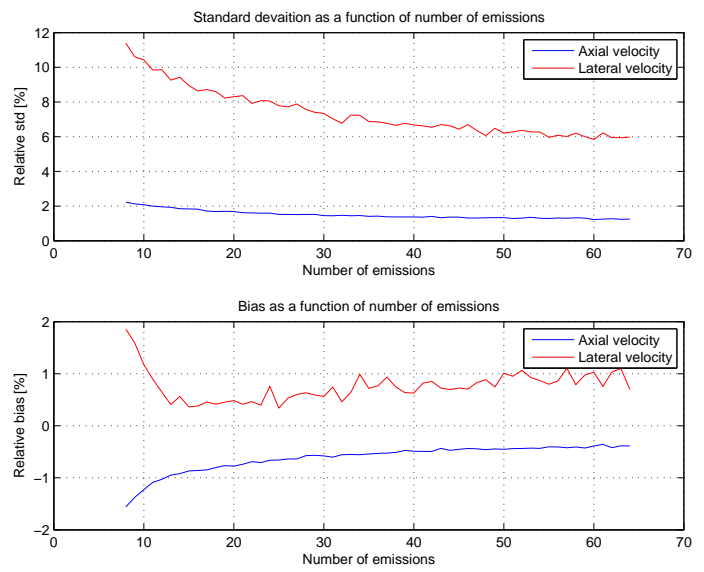

Fig. 7. Variation in the number of emissions for finding the velocity at a 75 degrees flow angle for the normalized standard deviation (top) and the bias (bottom) velocity component

velocity. For the axial component the mean standard deviation is $0.012 \mathrm{~m} / \mathrm{s}$ or $2.25 \%$ relative to the peak velocity. The mean standard deviation for the lateral velocity is $0.064 \mathrm{~m} / \mathrm{s}$ giving a relative standard deviation of $11.5 \%$. Compared to the true profile the mean bias for the axial velocity relative to the peak velocity is $-0.86 \%$ For the for lateral velocity it is $3.2 \%$. The performance as a function of number of lines used for the estimate is shown in Fig. 7 where the top graph shows the evolution in relative standard deviation and the lower graph in bias. Using the optimized lateral wavelength of $\lambda_{x}=1.55$ $\mathrm{mm}$ for the focusing and the resulting estimated wavelength of $1.61 \mathrm{~mm}$ in the estimate yields a bias that is less than $1 \%$ when employing more than 10 emissions. The resulting vector flow image is shown in Fig. 8, where the color intensity denotes velocity magnitude and the arrows indicate direction and magnitude. The beam-to-flow angle from this data was estimated to $91.9^{\circ} \pm 2.2^{\circ}$.

\section{CONCLUSION}

The TO approach can be extended to include convex array probes for a large field of view and thereby introduce vector

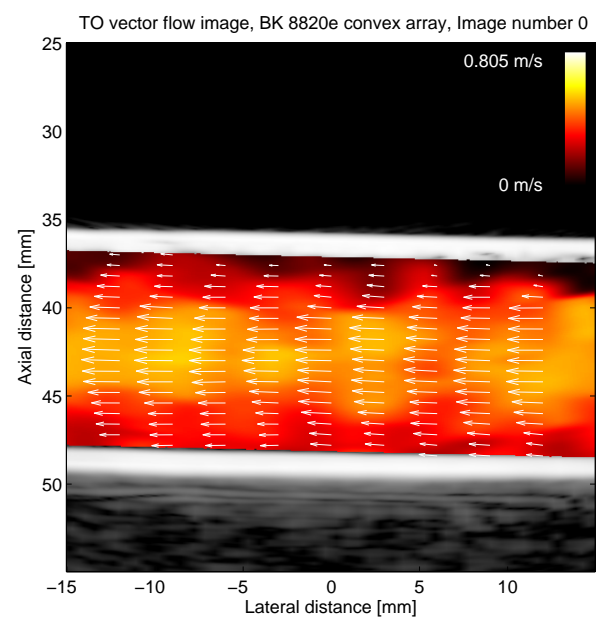

Fig. 8. Vector flow image measured on a flow rig phantom with a parabolic velocity profile and using the BK8820e convex array probe and the SARUS scanner.

flow imaging in the abdomen. The developed approach only uses 64 active elements during both transmit and receive and can readily be implemented on commercial platforms. Using 32 emissions a measured standard deviation of 5.6\% could be obtained for a fully transverse flow. Reducing the number of emissions to 16 increased the standard deviation to $8.4 \%$.The bias of the approach has been significantly reduces by optimizing the TO using simulations from $35 \%$ to around $0.5 \%$ for measurements in a flow rig.

\section{ACKNOWLEDGEMENT}

This work was supported by grant 024-2008-3 and grant 82-2012-4 from the Danish Advanced Technology Foundation and by B-K Medical Aps.

\section{REFERENCES}

[1] P. M. Hansen, M. M. Pedersen, K. L. Hansen, M. B. Nielsen, and J. A. Jensen, "Demonstration of a vector velocity technique," Ultraschall in Med, vol. 32, pp. 213-5, 2011.

[2] M. J. Pihl, J. Marcher, and J. A. Jensen, "Phased-array vector velocity estimation using transverse oscillations," IEEE Trans. Ultrason., Ferroelec., Freq. Contr, vol. 59, no. 12, pp. 2662-2675, December 2012.

[3] J. A. Jensen and P. Munk, "A New Method for Estimation of Velocity Vectors," IEEE Trans. Ultrason., Ferroelec., Freq. Contr., vol. 45, pp. 837-851, 1998.

[4] M. E. Anderson, "Multi-dimensional velocity estimation with ultrasound using spatial quadrature," IEEE Trans. Ultrason., Ferroelec., Freq. Contr., vol. 45 , pp. 852-861, 1998.

[5] J. A. Jensen, "A New Estimator for Vector Velocity Estimation," IEEE Trans. Ultrason., Ferroelec., Freq. Contr., vol. 48, no. 4, pp. 886-894, 2001.

[6] J. A. Jensen and N. B. Svendsen, "Calculation of Pressure Fields from Arbitrarily Shaped, Apodized, and Excited Ultrasound Transducers," IEEE Trans. Ultrason., Ferroelec., Freq. Contr., vol. 39, pp. 262-267, 1992.

[7] J. A. Jensen, "Field: A program for simulating ultrasound systems," Med. Biol. Eng. Comp., vol. 10th Nordic-Baltic Conference on Biomedical Imaging, Vol. 4, Supplement 1, Part 1, pp. 351-353, 1996.

[8] J. A. Jensen, H. Holten-Lund, R. T. Nilsson, M. Hansen, U. D. Larsen, R. P. Domsten, B. G. Tomov, M. B. Stuart, S. I. Nikolov, M. J. Pihl, Y. Du, J. H. Rasmussen, and M. F. Rasmussen, "SARUS: A synthetic aperture real-time ultrasound system," IEEE Trans. Ultrason., Ferroelec. Freq. Contr., p. In press, 2013. 